\title{
La tradición como mecanismo de inclusión y exclusión social
}

\author{
Ponce Alonso, Eduardo \\ Universidad Veracruzana, México \\ eduardoponcea@hotmail.com
}

Resumen - En el presente documento se expone una manera de acercamiento a los fenómenos de inclusión y exclusión desde el punto de vista de la tradición. El artículo muestra la manera como se ha entendido la tradición y propone un acercamiento como "tecnología social" desde la cual se entiende el funcionamiento antes que el contenido. Para evidenciar el funcionamiento de la tradición como tecnología social desde el enfoque de la inclusión y exclusión se retoma la etnografía de Sosme Campos realizada en la Sierra de Zongolica.

Palabras clave - Tradición; Tecnología social; Inclusión; Exclusión; Zongolica;

\begin{abstract}
This document presents a way of approaching the phenomena of inclusion and exclusion from the point of view of tradition. The article shows how tradition has been understood and proposes an approach as "social technology" from which operation is understood before content. To demonstrate the functioning of tradition as a social technology from the perspective of inclusion and exclusion, the ethnography of Sosme Campos, carried out in the Sierra de Zongolica, is taken as an example.
\end{abstract}

Keywords - Tradition; Social technology; Inclusion; Exclusion; Zongolica;

Interconectando Saberes, 2021

ISSN: 2448-8704

(cc) EY-NC-ND

\section{INTRODUCCIÓN}

El presente texto busca argumentar los mecanismos de inclusión y exclusión que los procesos de la tradición generan en los grupos sociales. Para lograrlo es necesario definir y conocer el funcionamiento de la tradición en grupos que, desde un contexto de abandono e inequidad, son considerados como marginados. Centraremos el análisis de este trabajo en los pueblos originarios para evidenciar que los mecanismos de inclusiónexclusión no sólo son generados por estructuras macrosociales sino también por circunstancias micro que pueden verse motivadas por la participación de actores externos.

En la edición anterior del presente libro, la idea de pobreza es el detonador de múltiples problemáticas que desembocan en inequidades, desigualdad y exclusiones. Hace ya varios años que las discusiones en torno al concepto de pobreza no se han centrado sólo en los aspectos económicos.

Fecha de Recepción: 18 de agosto de 2020

Fecha de Aceptación: 28 de enero de 2021 Fecha de Publicación: 31 de enero de 2021 
En los años recientes se ha consensado que es mejor hablar de pobrezas que de pobreza debido a la identificación de distintos tipos de esta: económicas, educativas, de salud, ciudadana, etc.

En ese sentido, el material que a continuación se presenta busca enriquecer las betas de reflexión al respecto de la inclusión y exclusión social y, de esta manera, no redundar en los datos ya aportados por otros investigadores. El documento se centra en evidenciar la construcción de la exclusión a partir de la tradición. El análisis que se presenta tiene como finalidad mostrar que, de manera intrínseca, los fenómenos de la tradición generan desigualdades al normalizar la realidad social. El funcionamiento de esas normas reguladoras de la vida social puede llegar a ser un mecanismo que, a veces de manera inconsciente, genera divisiones y exclusiones entre grupos de por sí ya vulnerables.

Bajo estas lógicas el texto busca mostrar a la tradición no como un fenómeno de folklorización cultural sino como un mecanismo intrínseco de las sociedades que permite generar sentido de pertenencia a sus integrantes. Desde este punto de vista, la tradición es el elemento que permitirá reflexionar sobre las ideas de inequidad, desigualdad y exclusión social como parte de las prácticas culturales de identidad de los diferentes grupos sociales. El principal aporte de este documento es evidenciar que el acceso a los recursos culturales generados por los procesos de la tradición no se da de manera equitativa y, por lo tanto, generan desigualdades y exclusiones.
Si bien es cierto que la idea de pobreza es, en muchas ocasiones, medida con criterios estadísticos que tienden más hacia lo cuantitativo, la base de los datos que se presentan para sostener las ideas de este documento apunta hacía el aspecto cualitativo lo cual no busca restar importancia a los análisis cuantitativos sino exponer desde otra perspectiva y con otros parámetros los fenómenos de la desigualdad y la exclusión.

Para lograr lo expuesto se ha estructurado un texto que va de las particularidades conceptuales de la idea de tradición, después se expondrá su funcionamiento en las realidades sociales y se concluye con un estudio de caso en donde se muestran los procesos de inclusión/exclusión a partir de los accesos a los recursos culturales generados por la tradición en el contexto de la Sierra de Zongolica.

\section{LÍMITES Y DEFINICIONES CONCEPTUALES DE LA TRADICIÓN}

Por lo general, la tradición se ha asociado a expresiones folclóricas de sociedades originarias. Desde perspectivas que han conceptualizado a la tradición se le ha relacionado con la manera de hacer cosas, de vestir, de comer, de vivir, como persistencias del pasado que configuran el presente 0 como legitimadores del orden establecido. Para los fines que este documento persigue es necesario distinguir entre tradición y tradiciones. Entendemos por tradición el proceso por medio del cual los grupos sociales transmiten saberes y conocimientos acumulados a través del 
tiempo; por lo tanto, las tradiciones son las expresiones culturales que surgen de los procesos de la tradición.

En ese sentido las tradiciones tienen un por qué y no sólo una finalidad lúdica u ociosa. En el contexto de pobreza, o pobrezas como se ha argumentado, pueden ser abordadas como estrategias y prácticas que los grupos sociales han desarrollado para sobrevivir los momentos de precariedad 0 exclusión económica, social y cultural a los que son expuestos.

Son varios los autores que han reflexionado y esbozado sobre la idea de tradición como mecanismo de inclusión/exclusión selectivo o bien de legitimador social. Robert Redfield, desde 1955, propuso la distinción entre la pequeña y la gran tradición. "I think we might begin with a recognition long present in discussions of civilizations of the difference between a Great Tradition and a Little Tradition" (Redfield, 1955: 14). Las aseveraciones que Redfield hace para sostener su distinción se basan en la dicotomía "Folk"-“Urbano" en la cual la pequeña y la gran tradición distinguen a sociedades o grupos con accesos a servicios y conocimientos diferenciados.

Una de las concepciones más trabajadas sobre la tradición es la desarrollada por Eric Hobsbawm quien escudriña la idea de "tradición inventada". Para Hobsbawm la tradición inventada implica lo siguiente:
El término tradición inventada se usa en un sentido amplio, pero no impreciso. Incluye tanto las "tradiciones" realmente inventadas, construidas y formalmente instituidas, como aquellas que emergen de un modo difícil de investigar durante un periodo breve $y$ mesurable, quizás durante unos pocos años, y se establecen con gran rapidez (Hobsbawm, 2012: 7).

Para Hobsbawm el objetivo y la característica de la tradición, inventada o no, es la invariabilidad; es decir, la tradición funciona como justificación del presente: una acción se justifica por su tradición. Así la tradición se remite a una cuestión normativa por lo cual cumple con una función de mecanismo sancionador y por lo tanto dictaminador de quien está dentro o fuera de lo permitido. El surgimiento de las tradiciones también responde a cuestiones prácticas. Las tradiciones se trasforman al momento que las normas vigentes se vuelven difíciles de seguir y de utilizar. Las nuevas tradiciones no se desligan de sus antecedentes, sino que son consecuencia de ellas.

La invención de la tradición, según Hobsbawn (2012:10), "es esencialmente un proceso de formalización y ritualización, caracterizado por la referencia al pasado, aunque sólo sea al imponer la repetición". Más allá de la formalización, la invención de tradiciones es "la invención de signos de pertenencia a un club cargados emocional y simbólicamente" (Hobsbawn, 2012: 17). La invención del signo busca generar consenso, colectividades y distinciones entre los grupos sociales. 
Desde otro ángulo, el antropólogo español Pedro Tomé ha argumentado que la idea de tradición debe ser puesta en debate no sólo por sus procesos y contenidos sino por los sujetos que buscan mantenerla y legitimarla. Pedro Tomé (2004: 57) menciona que el análisis correcto de las tradiciones "nos muestran cómo están operando en la sociedad actual procesos de prefiguración del futuro simultáneamente en procesos de constitución o consolidación de regiones culturales y de mundialización". El análisis que propone Tomé está enfocado en la identificación de los actores y principales impulsores de las tradiciones lo que lleva a abordarlo como elemento de dominación y en un entramado de relaciones de poder.

Thierry Linck le agrega un elemento importante a la idea de la tradición al reflexionarlo desde los fenómenos de la patriomonialización y su agregación de valor lo cual lo transforma en un recurso que se disputa. En este sentido, la tradición al transmitir saberes, conocimientos, reglas, valores, "patrimonios", se convierte en un "recurso complejo, dotado con atributos de bien colectivo y sin embargo sometido a rivalidades de uso" (Linck, 2011). Desde la propuesta de Linck, una vez que las expresiones tradiciones pasan por el proceso de patrimonialización deja de ser un bien libre al cual no cualquiera tendrá acceso.

Bajo la lupa que proponen estos pensadores, la tradición, además de ser un mecanismo que transmite ideas, saberes y conocimientos, funciona como una herramienta de regulación de la inclusión/exclusión en los grupos sociales a partir de las prácticas que implican las expresiones tradicionales. En ese sentido, los procesos de la tradición normalizan las acciones de inclusión y exclusión en los miembros de las comunidades.

Desde esta propuesta de conceptualización de la tradición no es un proceso inalterable e inamovible por lo cual es necesario analizarla y estudiarla como una práctica social que sirve para legitimar ciertos escenarios sociales en donde se certifican a sujetos con intenciones definidas quienes contribuyen a mantener la frontera del pertenecer 0 no a determinado grupo.

Otro punto importante es la agencia de los sujetos en los fenómenos de la tradición. La tradición como sistema de transmisión de información no se autorregula de manera autónoma, sino que son los integrantes del grupo a través del conocimiento y la experiencia trasmitida los que generan los cambios en las expresiones tradicionales. Desde este punto se pueden retomar las propuestas de "la tradición como reclamo" (Díaz, 2007) y "la tradición selectiva" (Williams, 1977).

Para Raymond Williams (1977: 137), el concepto de tradición debe ser entendido como una estructura selectiva, es decir, "una versión intencionalmente selectiva de un pasado configurativo y de un presente preconfigurado que resulta entonces poderosamente operativo dentro del proceso de definición e identificación cultural y social". Con esto, el autor deja en claro que la tradición, al ser considerada como mecanismo de selección, debe dejar de pensarse como 
supervivencia del pasado y ser abordada como un elemento configurador y seleccionador de experiencias. Williams abre el debate para pensar el concepto como un mecanismo de inclusión y exclusión a partir de la intencionalidad de los individuos para conformar una identidad y justificar o contrarrestar una hegemonía. Para Williams lo importante del estudio de las tradiciones es encontrar los puntos que permiten entender la conexión entre las experiencias seleccionadas del pasado para justificar el presente y, a partir de ahí, trazar las direcciones de futuro.

La idea que plantea Luís Díaz Viana va en el mismo tenor de selección y construcción que Williams. A partir de una crítica de la comercialización de la identidad, Díaz reflexiona sobre el cómo se construyen tradiciones para "exhibir lo exótico y proporcionar en los visitantes el consumo de la nostalgia; de reivindicar rutas de lo típico a partir de los vestigios de un "antes" -por lo demás bastante atemporal-; de proveer túneles con peaje para regresar a un pasado tan dudoso que -como talprobablemente jamás existió" (Díaz, 2007: 18). De lo que se trata es de seleccionar un pasado que permita construir una mercancía atractiva. Desde esta línea, la tradición se convierte en un recurso perfectamente aprovechable y vendible por "mediadores culturales", el problema aparece cuando dicha construcción ya no trata de "reivindicar lo propio, de defender la identidad cultural, como de adquirir mayor valor en el "mercado de lo distinto" para, así, lograr la más alta rentabilidad o exenciones fiscales" (Díaz, 2007: 26).
Desde las perspectivas esbozadas es conveniente retomar que las tradiciones son construcciones con intenciones. Los contenidos que se transmiten por la tradición no son neutros, sino que persiguen una finalidad: la construcción de identidades, la apropiación de elementos, la reducción de incertidumbres, la configuración presente, etc. Las experiencias que se transmiten son seleccionadas y transmitidas a partir de dos mecanismos específicos: la experiencia y la verbalización.

\section{“ASí ES LA TRADICIÓN". LOS MECANISMOS DE LA TRADICIÓN}

La tradición funciona y se reproduce por medio de la experiencia y la verbalización lo cual implica un cambio constante en los contenidos y expresiones tradiciones. En ese sentido la tradición tiene como motor principal la inclusión u olvido de nuevos elementos dependiendo de las circunstancias que el grupo afronta en su diario vivir. La experiencia es entonces lo que permite a los grupos balancear y poner a prueba los conocimientos que se tienen.

Sin embargo, a pesar de que muchas veces los tiempos y las circunstancias que se viven no encajan con los conocimientos que se tienen, las prácticas tradiciones se siguen reproduciendo no porque sean las más adecuadas sino porque guardan y conservan la estabilidad del orden social establecido. 
Son sujetos específicos (las monarquías, los patriarcas, los jerarcas, las elites, el pueblo) quienes muchas veces inculcan, proponen 0 "inventan" los elementos que se integran a los circuitos de la tradición.

La selección de experiencias no es lo único que posibilita el funcionamiento de la tradición. Retomando ideas de Gramsci hay sujetos "orgánicos" a los regímenes que se encargan de mantenerlo y justificarlo, pero también de transmitirlo a partir de la verbalización de la experiencia de vida del grupo social. Así es posible identificar a sujetos, o grupos de sujetos, que controlan el "buen desarrollo" de la tradición.

En ese sentido los cambios radicales en las expresiones tradiciones son entendidas como conflictos de intereses entre grupos que buscan conservar y los que buscan cambiar. Este tipo de cambios enfrenta a generaciones, géneros, grupos particulares y, más recientemente, a los exponentes de "la verdadera" tradición. Desde esta propuesta los fenómenos de patrimonialización que han venido a dotar de valor, no solo simbólico sino económico a las expresiones de la tradición, ha construido un campo de batalla por saber quién tiene o detenta la verdadera tradición cuando en realidad es un recurso que se construye de manera colectiva y por lo tanto se legitima en lo popular.

Lo paradójico de estos procesos es que en muchas ocasiones los que introducen los cambios 0 disputas por los recursos de la tradición no son personas asentadas en el grupo sino agentes que se encuentran en los límites sociales permitidos por el grupo en cuestión o por agentes externos que de manera específica tratan de romper con el orden establecido. Si bien es cierto que uno de los objetivos de la tradición es ser un elemento cohesionador de los grupos sociales, los agentes de cambio, tanto internos como externos, terminan por fragmentar y dividir a los integrantes.

El caso que a continuación se exponen enfatiza el proceso que se vivió cuando un agente externo incentivó los procesos de patrimonialización de una de las expresiones de la tradición lo cual, lejos de cohesionar entorno a los beneficios que significaba el proyecto, escindió y propició una disputa por un elemento que era un bien colectivo y que era aprovechado y explotado por todos los miembros del grupo.

\section{EXCLUSIÓN Y DESIGUALDAD EN LOS PROCESO DE LA TRADICIÓN}

Es necesario decir que en ciertos escenarios las expresiones de la tradición se construyen como respuesta a la exclusión a la que están expuestos lo grupos vulnerables. Sosme Campos (2015) da cuenta de la llegada de un agente externo que dinamiza los procesos de "empoderamiento" femenino entre las mujeres tejedoras de la Sierra de Zongolica, Ver, lo cual generó escisiones y divisiones dentro de las comunidades al dotar de valor un recurso de la tradición. Si bien es cierto que puede haber muchos casos que muestren lo que has ahora se ha argumentado, se ha elegido el ejemplo de las mujeres de Zongolica debido a la claridad con que el autor muestra cómo, a partir de 
una práctica tradicional que aparentemente carecía de valor, se generaron procesos de desigualdad, exclusión e incluso enemistadas al interior de un grupo de por sí ya vulnerable. ¿Quiénes y cuáles fueron los factores que hicieron de una práctica colectiva un mecanismo de exclusión y desigualdad social?

Algo importante de lo reportado por Sosme Campos es la realización del Proyecto de Rescate y Fomento Artesanal de la Sierra de Zongolica y la llegada de agentes externos. La llegada de "xinolah" (forastero) a las localidades de la Sierra de Zongolica dinamizaron los procesos de patrimonialización de las expresiones tradicionales vinculadas a la práctica textil. Así, Sosme menciona: "Aún por encima de los valores tradicionales, el grueso del grupo llegó a estar constituido por mujeres entre 30 y 35 años quienes por intercesión de las mayores lograron participar de la organización y las capacitaciones" (Sosme, 2015: 170). En estas circunstancias la intervención de los agentes externos no sólo dotó de valor comercial la práctica del tejido sino también a la organización social.

El proyecto organizó a las mujeres para incluirlas en los mercados estales y nacionales de los cuales habían estado ausentes o bien participado de manera secundaria y tangencial. La organización tenía como objetivo generar ingresos extras que ayudaran a mejorar las condiciones de vida de la población, pero con especial incidencia en la posición de la mujer en la localidad. Sin embargo, estos objetivos generaron también rupturas y escisiones al interior de los grupos.

Una de estas rupturas y enfrentamientos se dio entre géneros. Al dar una autonomía en el manejo de recursos económicos a las mujeres se confrontó con los roles de género establecidos. Debido a las actividades que desempeñaban las mujeres en las organizaciones parecía que se "descuidaban" las tareas que por tradición se habían asignado a las féminas: atención de los hijos, de los quehaceres domésticos, cuidado del esposo, permanecer en el espacio doméstico. Esta aparente falta de atención a las tareas femeninas motivo el enfrentamiento y el cuestionamiento por parte de los varones hacía la participación de sus esposas en los espacios y actividades del proyecto textil. Como bien señala Sosme, la verdadera razón de la confrontación era que con el ingreso de recursos económico por parte de las mujeres y el control que las mujeres tenían sobre el grupo se sentía como un atentado contra los cotos de poder establecidos y normalizados por la tradición.

La confrontación entre géneros no es lo único que provocó el proyecto de patrimonialización de las expresiones tradicionales textiles en la Sierra de Zongolica. Al agregar valor económico a expresiones que antes eran colectivas y con valor simbólico y cultural, los grupos que originalmente se organizaron para hacer frente a la exclusión de los mercados comenzaron a pugnar por su derecho legítimo de administrar lo que es "suyo". Sosme menciona lo siguiente: "Larios y su equipo abandonaron entonces la Sierra de Zongolica 
dejando atrás un sueño al fin cristalizado. Sin embargo, el tiempo y las circunstancias trazaron nuevos caminos en las vidas de las tejedoras que pronto terminaron uniéndolas o separándolas de por vida" (Sosme, 2015: 187).

El dejar a las tejedoras por su cuenta comenzó un proceso de inclusión y exclusión que se cimento en el manejo de los recursos como tejedoras y portadoras de una identidad étnica. La disputa por ese recurso dividió en más de una ocasión a los grupos y generó nuevos espacios en donde sólo determinados individuos podían acceder y desde donde podían negociar y legitimar un bien cultural. Así, mujeres que contaban con conocimiento en el ámbito textil pero que no podían acceder a las organizaciones de tejedoras quedaban fuera de los beneficios que como bien cultural se estaba explotando. Al respecto Sosme argumenta

Doña Manuela González es, por mucho, el más claro ejemplo de esta realidad triste y contrastante en la que el brillo de unas cuantas ha constituido el ocaso amargo de la mayoría. Así, los cuatro premios nacionales que le han proyectado a la escena nacional (aun sin saber tejer) no se explican sin la explotación de las mujeres que por su trabajo recibieron 200 pesos y una bolsa de pan (Sosme, 2015: 310).

El trabajo de los agentes externos que en un principio logró y motivó el "empoderamiento" de algunas de las mujeres de la zona de trabajo ocasionó la exclusión y desigualdad de acceso a los beneficios que por años se habían estado construyendo como un recurso colectivo. La apropiación de los mecanismos de la tradición, y más aún de las expresiones tradicionales, generó tanto al interior como al exterior del grupo accesos desiguales a los conocimientos y recursos políticos y económicos que emanaron del proyecto.

Bajo estas perspectivas los fenómenos de la tradición, ya sea en sus procesos como en su contenido, no sólo generan una distinción entre un adentro y un afuera, sino que de manera intrínseca es un mecanismo de regulación de desigualdad, inclusión y exclusión social.

El retrato que la etnografía de Sosme nos proporciona nos permite evidenciar que muchas veces agentes externos reflexionan la tradición como mecanismo neutro lo cual puede llegar a soslayar las diferencias, pero llega a abrir muchas más. La tradición como mecanismo de diferenciación permite entender que los procesos de exclusión no sólo son externos a las sociedades, sino que se pueden registrar y generar en su propio funcionamiento.

\section{CONCLUSIONES}

El presente documento evidencia una arista más de la desigualdad que se genera debido a los fenómenos de inclusión y exclusión. Para hacerlo, nos hemos valido del marco de la tradición y su conceptualización lo cual permite ver otro escenario a los ya planteados por otros autores.

Algo que buscamos resaltar con el estudio de caso es la participación de agentes externos (ONG o instancias oficiales) como generadoras desigualdad y por lo tanto de inclusión y exclusión 
social. En algunas de las ocasiones la participación de organismos externos puede ayudar a contra restar los efectos de lo exclusión macroestructural, pero en algunas circunstancias genera otras al "empoderar" a sujetos locales que articulan fenómenos de impacto local.

Es en ese sentido que argumentamos y mostramos cómo la tradición es un proceso y mecanismo manipulable y manipulador. La tradición puede ser manipulada en beneficio de alguno de los grupos sociales y, por el otro lado, al ser regulador del orden social y muchas incuestionable, "siempre se ha hecho así" o "así es la tradición", llega a ser un elemento manipulador del comportamiento y orden sociales establecidos. Sin embargo, una vez que los mecanismos de la tradición se hacen evidentes quienes se los apropian los pueden llegar a utilizar como herramientas de distinción y de contestación contra hegemónica tal y como lo han evidenciado otros procesos como puede ser el fenómeno del Túmin en otra parte de la geografía veracruzana.

Estas reflexiones apuntan e invitan a los lectores a ver y a analizar los aspectos de inequidad, desigualdad, exclusión e inclusión desde los aspectos micro y subjetivos y no sólo desde los aspectos macro y objetivos.

\section{REFERENCIAS}

Díaz Viana, L., (2007). Antropología y patrimonio cultural: la tradición como reclamo, en Díaz Viana, Luís y Pedro Tomé (Coord.) (2007), La tradición como reclamo. Antropología en Castilla y León, Junta de Castilla y
León/consejería de cultura y turismo, España, pp. 17-30.

Hobsbawm, E. \& Terece, R., (2012). La invención de la tradición, Crítica, España, 318 pág.

Linck, T., (2011). Del patrimonio a la patrimonialización en Linck, Thierry, Julio Moguel y Alfredo Ramírez, 2011, Economía popular y procesos de patrimonialización, México, Juan Pablos Editor / Fundación México social siglo XXI, México.

Redfield, R., (1955), The social organization of tradition. The far Eastern quarterly, vol. 15, No. 1(Nov. 1955), pp. 13 -21.

Sosme Campos, M. Á., (2015), Tejedoras de esperanza. Empoderamiento en los grupos artesanales de la Sierra de Zongolica, Colmich, México, 336 pág.

Tomé, P., (2004), Ecología cultural y procesos económicos en la conformación de las regiones culturales, en Regiones Culturales/Culturas regionales, CONACULTA/CONECULTA-Querétaro/EI Colegio de San Luís, México, pp. 51-67.

Williams, R., (1977). Marxismo y literatura, Ediciones península, Barcelona, 250 pág. 